\title{
GUIDELINES FOR THE MANAGEMENT OF HIV-INFECTED CHILDREN (NATIONAL DEPARTMENT OF HEALTH, SOUTH AFRICA 2005)
}

\section{A summary of antiretroviral treatment guidelines}

\author{
Tammy Meyers, FCPaed (SA) \\ Harriet Shezi Children's Clinic, Chris Hani Baragwanath Hospital, fohannesburg \\ Brian Eley, FCPaed ( $S A$ ) \\ Red Cross Children's Hospital, Cape Town
}

National guidelines for the management of HIV-infected children in South Africa have been developed as a consensus document by practising HIV clinicians around the country. Guidelines for antiretroviral (ARV) management of children appear in a booklet distributed by the National Department of Health (DoH) since mid-2004 (National Antiretroviral Treatment Programme Guideline for Carers, National Department of Health, South Africa, 2004). A comprehensive booklet on management of HIV-infected children has also been produced for the DoH which includes recommendations on management with antiretroviral (ARV) therapy.

The South African national paediatric guidelines are based on the recommendations provided by the World Health Organization (WHO) (Scaling up antiretroviral therapy in resource limited settings; treatment guidelines for a public health approach WHO 2003). It is important to note that WHO are updating their recommendations. Based on this some amendments, e.g. the staging system, have already been incorporated into the final version of the South African national guidelines. Other recommendations are more recent and have yet to undergo broader review. These have therefore not formed part of the printed version of the South African guidelines. It is anticipated that a process of updating the guidelines needs to be put in place urgently. The national guidelines are available on the government website (www.health.gov.za), and clinicians are advised to update themselves regularly at this website for any amendments that may be made to the guidelines.

It is essential that clinicians involved with management of HIVinfected children in both the private and the public sector are familiar with national recommendations for treatment. The majority of HIV-infected children will access care in the public sector and many move between private and public sector. Treatment choices made by private clinicians should take into account what is available in the public service, particularly if the patient is likely to access care from both sectors.

Owing to the scale of the HIV epidemic, and in order to reach all those who need care, people need to have access to comprehensive HIV care at health care facilities as close as possible to their homes. Uncomplicated cases, both adult and paediatric, should be managed at primary care facilities. The South African national guidelines are designed for clinicians practising at all levels of care. In many cases primary health care nurses may need to commence and manage children and adults on ARVs. Since ARV treatment is uncomplicated in most cases, this should be well within the scope of practice of appropriately clinically trained nurses.

\section{GOALS OF ANTIRETROVIRAL THERAPY}

The goals of ARV therapy for children are to increase survival, improve quality of life and decrease HIV-related morbidity and mortality.

The child's CD4 count should rise and remain above the baseline count.

- The child's viral load should become undetectable $(<400$ copies/ml) and remain undetectable on ARV therapy, although in some children a suppressed though detectable viral load, with sustained elevation in CD4 count and absence of intercurrent and/or opportunistic infection, may be the best achievable goal.

\section{ELIGIBILITY FOR ANTIRETROVIRAL THERAPY}

Patients must satisfy clinical and social criteria before being accepted for treatment.

\section{CLINICAL CRITERIA}

1. Confirmation of diagnosis of HIV infection.

2. Recurrent (>2 admissions per year) hospitalisations for HIV complications or prolonged hospitalisation for HIV (> 4 weeks), or

3. The patient satisfies the provisional WHO citeria for stage III/IV disease (see Interim Revised WHO Staging of Disease) or

4. For relatively asymptomatic patients, one can consider starting ARV if the CD4 percentage is $<20 \%$ (age under 18 months) or $<15 \%$ (age over 18 months). 


\section{SOCIAL CRITERIA}

These criteria are extremely important for the success of the programme and need to be adhered to. The principle is that adherence to treatment must be at least probable.

1. At least one identifiable caregiver who is able to supervise the child for administering medication (orphans and vulnerable children (OVC) should not be discriminated against and all efforts should be made to ensure that their psychosocial circumstances be addressed so that they too can receive treatment).

2. Disclosure to another adult living in the same house is encouraged (but not mandatory), so that there is someone else who may assist with the child's ART.

3. NB: Treatment of mother/caregiver/other family members

Always ask about the caregiver's health, and the health of other members of the family.

Ensure that mothers and other family members access medical care timeously, including ART if needed.

Where possible, HIV-positive mothers and caregivers requiring medical attention should be attended to at the same time as their children in order to decrease the number of clinic visits and associated costs and time away from work.

\section{WHAT TO START?}

Antiretroviral drug choices for children are set out in Table I.

\begin{tabular}{|c|c|c|}
\hline \multicolumn{3}{|c|}{$\begin{array}{l}\text { TABLE I. ANTIRETROVIRAL DRUG CHOICES FOR } \\
\text { CHILDREN }\end{array}$} \\
\hline & 6 months up to 3 years & $>3$ years and $>10 \mathrm{~kg}$ \\
\hline 1st line & $\begin{array}{l}\text { Stavudine (d4T) } \\
\text { Lamivudine (3TC) } \\
\text { Kaletra }^{\circledR}\end{array}$ & $\begin{array}{l}\text { Stavudine (d4T) } \\
\text { Lamivudine (3TC) } \\
\text { Efavirenz }\end{array}$ \\
\hline 2nd line & $\begin{array}{l}\text { Zidovudine (AZT) } \\
\text { Didanosine (ddl) } \\
\text { Nevirapine/efavirenz* }\end{array}$ & $\begin{array}{l}\text { Zidovudine (AZT) } \\
\text { Didanosine (ddl) } \\
\text { Kaletra }^{\circledR}\end{array}$ \\
\hline
\end{tabular}

\section{GENERAL COMMENTS}

All infants under 6 months of age who require ARV therapy should preferably be started on treatment under specialist supervision.

- Stavudine solution requires refrigeration. If no fridge is available, stavudine capsules may be opened and dissolved, and the required amount administered to the child. The rest can be discarded. If the caregiver experiences difficulty with stavudine capsules, zidovudine suspension may be used instead.

- Switch to tablets or capsules from syrups or solutions as soon as possible.

- Lopinavir/ritonavir (Kaletra ${ }^{\circledR}$ ) needs to be kept cool $\left(<25^{\circ} \mathrm{C}\right)$.
Didanosine (ddl) must be taken alone, on an empty stomach, at least an hour before (or 2 hours after) a meal. Tablets should be dissolved in at least $30 \mathrm{ml}$ of water. It is important to use 2 tablets of didanosine to obtain sufficient antacid buffering, e.g. if the child needs $100 \mathrm{mg}$ prescribe $2 \times 50 \mathrm{mg}$ tablets.

- Children may occasionally need to change from a drug in the first-line regimen to one from the second-line regimen, because of intolerance or a serious adverse reaction. Swapping limits the patient's second-line treatment options. The decision to swap must be made by a doctor with ARV experience. Swapping of one drug should only be done if there is full viral suppression, failing which the whole regimen may need to be altered. If intolerance develops to ritonavir or lopinavir/ritonavir (Kaletra ${ }^{\circledR}$ ), switch to nelfinavir. Abacavir may be used if adverse events on other nucleoside reverse transcriptase inhibitors (NRTIs) occur.

Drugs not listed in the first- and second-line regimens but mentioned in the guidelines, e.g. ritonavir, nelfinivir, saquinavir, abacavir and nevirapine, should be available at all tertiary care centres.

DoH guidelines have incorporated a dosing table that has been based on weight bands in order to facilitate ease of prescribing paediatric formulations (Table II).

\section{ADVERSE EVENTS}

The adverse event profile of ARVs is similar in adults and children. Most side-effects of treatment are minor if they occur, but on occasion severe adverse events may require urgent referral to centres with expertise and ARVs may need to be stopped. Such events include severe hepatitis, StevensJohnson syndrome, pancreatitis, lactic acidosis (presenting with severe gastrointestinal symptoms, pain, vomiting and/or decreased level of consciousness). Refer to 'Guidelines for management of the HIV-infected child' (www.health.gov.za) for more detailed information regarding adverse events.

Children on stavudine may, like adults, develop peripheral neuropathy and/or lipodystrophy/lymphoatrophy. These may be subtle or more difficult to diagnose in children. Nevertheless, a high index of suspicion may result in more adverse events being detected in children in general. Switching from stavudine to abacavir may be helpful if the child has viral suppression.

Remember that all adverse events should be notified to the Medicines Control Council (MCC). A copy of the yellow form that is required to be completed may be found in the full guidelines and/or is obtainable from the MCC offices.

\section{ADHERENCE}

Adherence greater than 95\% will ensure a good virological response and prevent the emergence of viral resistance. Good adherence can be achieved with regular education and support. Adherence may be monitored using diary cards, medication check and other measures. All efforts should be made to encourage this level of adherence. 


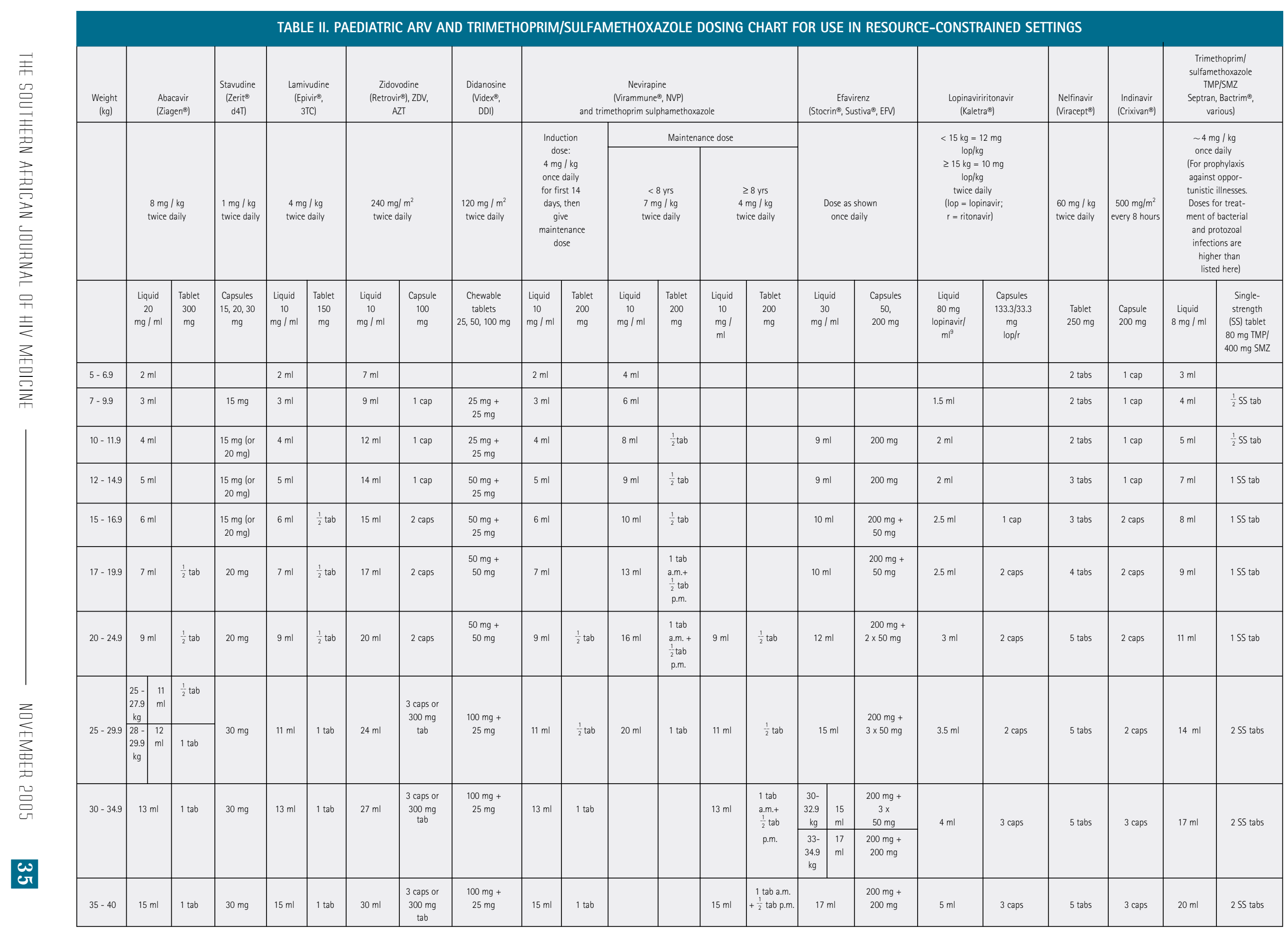


It is not possible for health care providers to reliably predict which caregivers or individuals will ultimately be adherent to their treatment plan, as adherence does not correlate with gender, cultural background, socioeconomic or education level, or language barriers between provider and patient. It is therefore essential to provide all caregivers with a comprehensive plan to support adherence. Several strategies need to be applied and all members of the health care team, as well as family and possibly even community-based support groups, need to be involved.

Experience has shown that adherence decreases as time progresses. Monitoring and support of adherence is therefore essential. Some factors affecting adherence to paediatric ART are listed below. A trusting relationship between the patient and caregiver and members of the health care team is essential. Optimal adherence requires full participation by the health-care team.

Every interaction with the patient and caregiver provides an opportunity for reinforcing the absolute need for adherence.

\section{SOME IMPORTANT FACTORS DIMINISHING ADHERENCE IN CHILDREN}

- Unanticipated drug side-effects and adverse events.

- Intercurrent illness.

- Caregiver illness/death or otherwise occupied.

- Patient resistance to taking medicines - ritonavir, singly or in combination with lopinavir, has a very bitter taste.

- Change or absence of patient's nurse or doctor.

- Frequent daily doses - e.g. twice or three times per day.

NB: Every effort should be made to ensure that every child who needs ARV can access this regardless of home circumstances. If necessary involve counsellors, social workers or even the school to ensure that the child may be adherent to his/her ARV therapy. In even the worst social circumstances a creative solution can and must be found.

\section{SWITCHING TO SECOND-LINE THERAPY}

Reasons for switching to second-line therapy are set out in Table III.

N.B.: Changing from first- to second-line ARV is a decision that is undertaken only after careful consideration. It is not something that one would rush into before considering possible improvements in managing therapy at home.

First check adherence: if it is not possible to improve adherence, attempt directly observed therapy (DOT) with a health care worker or the trusted 'other' family member or friend identified under 'social criteria' above.

It is essential that switching ARV regimens is done in consultation with a paediatrician with ARV experience.
TABLE III. REASONS TO MOVE TO SECOND-LINE ARV THERAPY IN CHILDREN

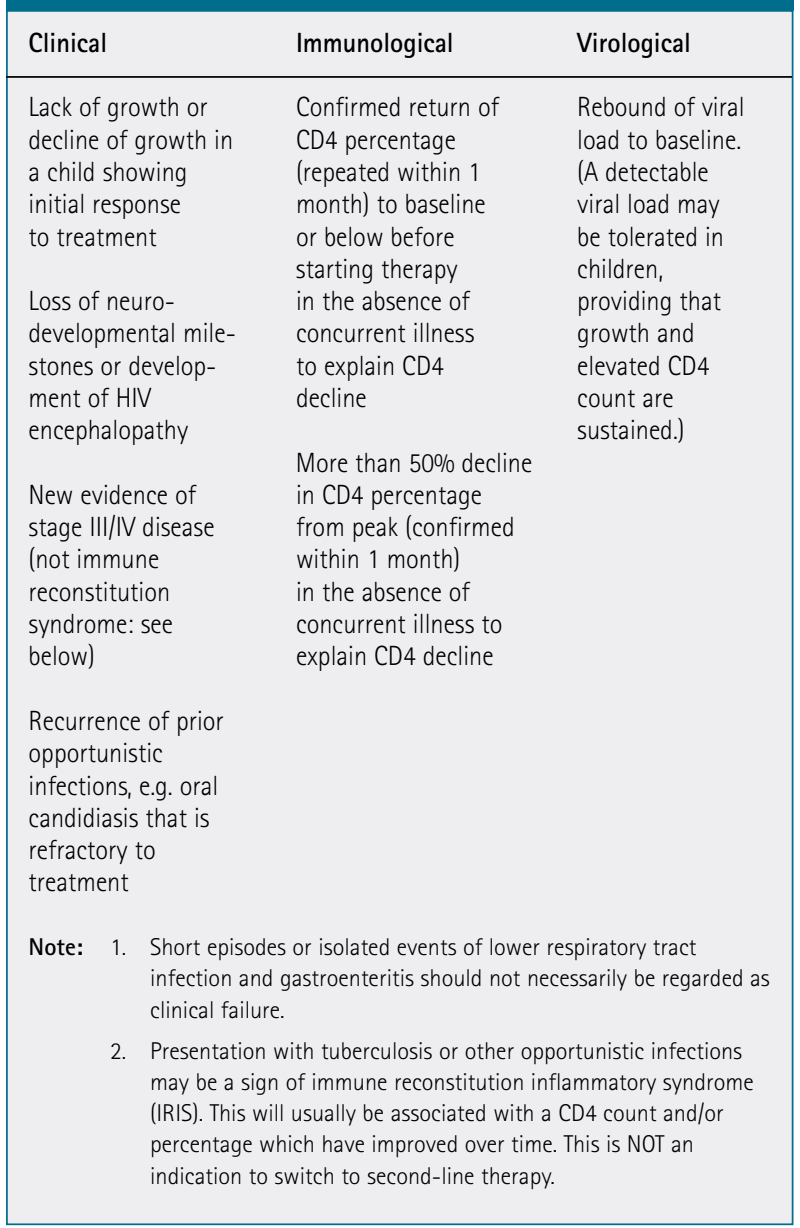

\section{CONCOMITANT TUBERCULOSIS}

Tuberculosis occurs commonly with HIV. There are two scenarios to consider:

1. Child presents with tuberculosis before commencing ARV therapy.

- Complete TB therapy if possible before commencing ARV therapy or delay ART for at least 2 months (in severe cases it may be necessary to commence ARVs even sooner).

- If the child has failed the nevirapine vertical transmission programme or is less than 3 years old or weighs $<10 \mathrm{~kg}$, use ritonavir as the third drug.

- If the child was not on the nevirapine vertical transmission programme and is more than 3 years old and weighs more than $10 \mathrm{~kg}$, use efavirenz as the third drug (monitor alanine transaminase (ALT) monthly).

2. Child develops tuberculosis while on ARV therapy.

- If the child is on lopinavir/ritonavir or nelfinavir, then switch to ritonavir.

If the child is on nevirapine, and is less than 3 years old or weighs less than $10 \mathrm{~kg}$, switch to ritonavir.

- If the child is on nevirapine and is more than 3 years old and weighs more than $10 \mathrm{~kg}$, switch to efavirenz.

- If the child is unable to tolerate the large number of drugs, 
ARV therapy may have to be interrupted until TB therapy has been completed (always discuss this with an experienced paediatric HIV clinician).

\section{Monitor ALT monthly.}

Note: Discuss all cases with a paediatrician with ARV experience, before interrupting therapy.

\section{MONITORING}

See Table IV.

\section{CLINICAL STAGING}

See Table V.

\begin{tabular}{lll|}
\multicolumn{3}{|l|}{ TABLE IV. PAEDIATRIC ARV REGIMENS AND ROUTINE } \\
MONITORING DURING TREATMENT
\end{tabular}

We would like to acknowledge all HIV clinicians around the country who assisted with the development of the above guidelines.
TABLE V. INTERIM REVISED WHO CLINICAL STAGING OF HIV/AIDS FOR INFANTS AND CHILDREN*

\section{Clinical stage 1}

Asymptomatic

Persistent generalised lymphadenopathy

\section{Clinical stage 2}

Hepatosplenomegaly

Papular pruritic eruptions

Seborrhoeic dermatitis

Extensive human papillomavirus infection

Extensive molluscum contagiosum

Fungal nail infections

Recurrent oral ulcerations

Lineal gingival erythema (LGE)

Angular cheilitis

Parotid enlargement

Herpes zoster

Recurrent or chronic respiratory tract infections (otitis media, otorrhoea, sinusitis)

\section{Clinical stage 3}

Moderate unexplained malnutrition not adequately responding to standard therapy

Unexplained persistent diarrhoea (14 days or more)

Unexplained persistent fever (intermittent or constant, for longer than 1 month)

Oral candidiasis (outside neonatal period )

Oral hairy leukoplakia

Acute necrotising ulcerative gingivitis/periodontitis

Pulmonary tuberculosis

Severe recurrent presumed bacterial pneumonia

Unexplained anaemia $(<8 \mathrm{~g} / \mathrm{dl})$, and/or neutropenia

$\left(<500 / \mathrm{mm}^{3}\right)$ and/or thrombocytopenia $\left(<50000 / \mathrm{mm}^{3}\right)$ for more than 1 month

Chronic HIV-associated lung disease including bronchiectasis Symptomatic lymphoid interstitial pneumonitis (LIP)

\section{Clinical stage 4}

Unexplained severe wasting or severe malnutrition not adequately responding to standard therapy

Pneumocystis pneumonia

Recurrent severe presumed bacterial infections (e.g. empyema, pyomyositis, bone or joint infection, meningitis, but excluding pneumonia)

Chronic herpes simplex infection (orolabial or cutaneous of more than 1 month's duration)

Extrapulmonary TB

Kaposi's sarcoma

Oesophageal candidiasis

CNS toxoplasmosis (outside the neonatal period)

HIV encephalopathy

Cytomegalovirus infection (CMV retinitis or infection of organs other than liver, spleen or lymph nodes; onset at age 1 month or more)

Extrapulmonary cryptococcosis including meningitis

Any disseminated endemic mycosis (e.g. extrapulmonary

histoplasmosis, coccidiomycosis, penicilliosis)

Cryptosporidiosis

Isosporiasis

Disseminated non-tuberculous mycobacterial infection

Candida of trachea, bronchi or lungs

Visceral herpes simplex infection

Acquired HIV-associated rectal fistula

Cerebral or B-cell non-Hodgkin's lymphoma

Progressive multifocal leukoencephalopathy (PML)

HIV-associated cardiomyopathy or HIV-associated nephropathy

*For persons aged under 15 years with confirmed laboratory evidence of HIV infection; HIV antibody if aged 18 months and above; virological or p24 antigen testing if aged under 18 months. 